\title{
Combien valent les décès évités par la prévention ?*
}

\author{
Olivier Chanel ${ }^{\dagger}$ et Jean-Christophe Vergnaud ${ }^{\ddagger}$
}

\begin{abstract}
A bstract
Cet article propose une méthode d'évaluation monétaire des politiques de prévention dont les bénéfices sanitaires s'étalent dans le temps. La méthodologie proposée prend en compte l'effet de latence entre la mise en place de la politique et l'obtention de l'ensemble des bénéfices sanitaires relatifs à la mortalité. Ces bénéfices sont évalués en termes d'années de vie gagnées et de décès prématurés évités pour deux programmes de prévention : une réduction de la pollution de l'air et une politique de prévention routière. Les principaux enseignements sont que la comparaison relative des bénéfices des deux politiques s'avère très sensible aux choix de la technique de monétarisation et du taux d'actualisation.
\end{abstract}

\section{How much are deaths avoided by the prevention worth?}

\section{A bstract}

This paper concerns the difficulty of taking into account long term health effects of prevention policies in an economic valuation. The proposed methodology estimates the delay between the implementation of a policy and the achievement of all of the expected mortality-related

\footnotetext{
${ }^{*}$ Les auteurs tiennent à remercier deux rapporteurs anonymes de la revue pour leurs suggestions et commentaires pertinents.

${ }^{\dagger}$ CNRS-GREQAM-IDEP, 2 Rue de la Charité, 13002 Marseille, France, Tel. : + 33 (0)491 140 780; courriel : chanel@ehess.univ-mrs.fr.

${ }^{\ddagger}$ CNRS-EUREQua, 106-112 Bd de l’Hôpital, 75647 Paris Cedex 13, France, Tél : +33

(0)144078 229; courriel : vergnaud@univ-paris1.fr.
} 
health benefits. Benefits are assessed both in terms of life years gained and premature deaths avoided for two prevention policies: an air pollution abatement policy and a road safety policy. The main findings are that the relative comparison of the policies' benefits is very sensitive to technical choices dealing with the method chosen to assess mortality benefits and the discount rate.

Classification J EL : H43, I12, Q25 


\section{Introduction}

L'estimation des bénéfices associés à une réduction de la mortalité constitue un enjeu important dans la recherche de politiques de prévention optimales, notamment dans les champs de la santé et de l'environnement qui l'abordent souvent de façon différente.

En économie de la santé, l'analyse en termes d'années de vie gagnées est la plus couramment employée, en particulier du fait de la meilleure caractérisation du bien "année de vie" que du bien "vie" lui-même. Le plus souvent, les résultats sont présentés en termes de ratio coût-efficacité ou coût-utilité, donnant un ordre de priorité sur les politiques à mener. Respecter cet ordre de priorité guide alors le décideur public vers l'efficience : la maximisation du nombre d'années de vie gagnées, éventuellement pondérées par leur qualité (sur les Quality-adjusted life years (QALY), voir par exemple Pliskin et al., 1980; Loomes et McKenzie, 1989; ou Bleichrodt, 1995).

En économie de l'environnement, l'approche privilégiée est celle de l'analyse coûts-bénéfices et réclame une monétarisation explicite des gains sanitaires. Lorsque la mortalité est prise en compte dans les études d'impacts sanitaires, l'approche en termes de décès (prématurés) évités, qui nécessite un recours à une Valeur d'Evitement d'un Décès (VED) est généralement adoptée.

Les approches coût-efficacité et coûts-bénéfices ne conduisent donc pas nécessairement à des recommandations identiques. Comme le remarque Hammitt (2003), une approche en terme d'années de vie gagnées, et plus particulièrement l'approche QALY, restreint la prise en compte des préférences individuelles aux seules longévité et qualité de vie, alors que l'analyse coûtsbénéfices, lorsqu'elle se fonde sur une évaluation des consentements à payer (CAP), tient totalement compte des préférences individuelles. Même si les fondements de l'économie du bien-être militent pour la seconde, Hammitt (2003) note que l'approche coût-efficacité est peut-être plus à même de tenir compte de dimensions supplémentaires présentes dans les préférences sociales (éthique, altruisme...), et reflète de ce fait mieux les perceptions sociales.

Dans tous les cas, le décideur public sera confronté à un arbitrage entre 
efficience et équité, puisque les préférences collectives en matière de santé ne répondent pas forcément à un critère de justice sociale, qui reste par ailleurs à préciser (voir Lindholm et al., 1996; Moatti, 1997; Ubel et al., 2000 ou Kaplan et Merson, 2002). Ainsi, pour se cantonner à une seule dimension, l'âge de décès intervient dans la valorisation économique, quelle que soit l'unité de mesure sanitaire de la mortalité : années de vie ou décès prématurés. L'évitement du décès d'un enfant et celui d'une personne âgée correspondent-ils à la même valeur économique? Cette question est cruciale si l'on souhaite qu'il revienne plutôt au calcul économique qu'à la seule pression de l'actualité d'éclairer le décideur public sur ses priorités en matière de programmes de santé. ${ }^{1}$

De nombreuses approches attribuant une valeur à la vie humaine la font dépendre de l'âge de la personne sauvée, et la forme de cette dépendance peut fortement différer. L'approche comptabilisant des années de vie gagnées générera logiquement une plus grande valeur économique lorsque les personnes sauvées sont jeunes. En revanche, les méthodes fondées sur la révélation de CAP obtiennent les valeurs maximales pour des âges de décès entre 40 et 50 ans (voir Shepard et Zeckhauser, 1982; Regens, 1991; Johannesson et Johansson, 1996, 1997; ou Krupnick et al., 2002).

La problématique se complexifie encore lorsque les politiques de prévention intègrent des facteurs de risque présentant un effet de latence, c'est-à-dire un délai s'écoulant entre la mise en oeuvre et l'observation de la réduction de la mortalité. Ignorer cet effet de latence conduit à une estimation incorrecte des bénéfices d'une politique de prévention, tant en termes sanitaires que monétaires, qui peut donc générer des inefficacités dans l'allocation de ressources dévolues à l'amélioration de l'état de santé d'une population.

L'objectif principal de ce papier est de proposer une méthodologie qui permette la prise en compte correcte de l'effet de latence, que ce soit dans le cadre d'une analyse en termes d'années de vie gagnées ou de décès prématurés évités. Elle nécessite une analyse temporelle des bénéfices et étudie plusieurs méthodes de valorisation économique. A titre d'application, deux politiques de prévention sont comparées : l'une visant à réduire la mortalité attribuée à l'exposition à la pollution atmosphérique et l'autre aux accidents de la route. 
Bien que la première se traduise par des effets de court et de long terme alors que la seconde n'implique que des effets de court terme, les enjeux sanitaires de ces deux politiques sont comparables.

Ainsi, les accidents de la route ont été responsables de 8.080 décès en 1996, survenus à l'âge moyen de 39 ans environ. Chacun correspondait à une perte d'espérance de vie de 41 ans, soit un total 331.300 années de vie perdues annuellement. La même année, Künzli et al. (2000) attribuent 31.692 décès annuels aux effets de la pollution de l'air particulaire, dont la moitié environ est due aux émissions du trafic routier. L'âge moyen des décès prématurés est plus élevé, de l'ordre de 77 ans, ce qui représente une perte d'environ 10 ans d'espérance de vie à cet âge, soit un chiffre comparable de 316.900 années de vie perdues chaque année. ${ }^{2}$ Le choix de la monétarisation de la mortalité est crucial : choisir une VED indépendante de l'âge de décès renforce par exemple grandement l'intérêt relatif d'une politique de réduction de l'exposition à la pollution atmosphérique par rapport à celle d'une politique de prévention routière.

La seconde section propose un cadre méthodologique pour estimer l'évolution temporelle des taux de mortalité en présence d'un effet de latence, et présente les méthode de monétarisation de la mortalité retenues. La troisième section concerne l'application de la méthodologie aux deux politiques de prévention choisies. Après avoir étudié l'importance de l'effet de latence, nous comparons l'intérêt relatif des deux politiques selon le choix retenu de monétarisation et selon le niveau du taux d'actualisation.

\section{M éthodologie}

Considérons un décideur public désirant améliorer l'état sanitaire d'une population en mettant en place une politique de prévention primaire agissant sur un facteur de risque sanitaire. Cette politique est susceptible de générer des effets à court et à long terme, qu'il faut quantifier au sein d'une approche formalisée avant de les transformer en valeur monétaire. 


\subsection{Cadre épidémiologique}

\subsubsection{Politique de prévention et risque relatif}

Le concept de Risque Relatif $(R R$,$) constitue le point de départ de l'analyse,$ et se définit comme le risque, chez une population exposée à un facteur causal, d'être affecté par un événement $\left(R^{E}\right)$, divisé par le même risque chez une population non exposée $\left(R^{N E}\right)$. Ce concept s'applique aussi bien à des effets de court terme (instantanés, tels les accidents de la route) qu'à des effets de long terme (qui surviennent plusieurs années après l'exposition au facteur causal, comme les cancers du poumon). L'hétérogénéité de l'exposition et/ou la sensibilité de la population à un facteur donné se traduisent pas une dépendance des $R R$ à certaines caractéristiques de la population. Soit donc $R R_{p}^{E}=\frac{R_{p}^{\mathrm{E}}}{R_{\mathrm{p}}^{\mathrm{E}}}$ le $R R$ d'une population caractérisée par $p$ et soumise à une exposition de niveau $E$.

Considérons une politique de prévention qui diminue l'exposition au facteur de risque d'une population de caractéristiques $p$. Sa durée est de $\Delta$ années, avec $\Delta \in\{1,2, \ldots\}$, la politique étant qualifiée de définitive pour $\Delta=\infty{ }^{3}$ D'une façon générale, cette politique va agir sur l'évolution du $R R$ de la façon suivante :

$$
R R_{p, t}=g(E, \Delta, p, t ; \tau)
$$

où

- $R R_{p, t}$ représente le $R R$ de la population caractérisée par $p, t$ années après la mise en place de la politique de prévention,

- E représente le niveau initial d'exposition,

- $\Delta$ représente la durée pendant laquelle la politique est mise en oeuvre,

- $\tau$ caractérise l'évolution de $R R_{p, t}$ en fonction du délai écoulé depuis la mise en place de la politique,

- $g(. ; \tau)$ est une forme fonctionnelle, avec $\partial g(. ; \tau) / \partial t<0$ si $t<\Delta$ et $\partial g(. ; \tau) / \partial E>0$. 
La forme de la fonction $g(. ; \tau)$ dépendra en particulier de l'importance de la période de latence entre l'exposition passée et les conséquences sanitaires, de la façon dont le niveau de risque va diminuer consécutivement à la diminution de l'exposition et des caractéristiques de la population concernée par la politique de prévention (fumeurs, femmes enceintes, enfants...).

Ainsi, dans le cas des accidents de la route, le $R R$ va principalement dépendre de l'âge et du sexe, et ne va pas faire intervenir de temps de latence. En effet, dès lors qu'un individu évite un décès (ou une blessure) causé par un accident de la route, sa probabilité de décès (ou son état de santé) est semblable à celle de tout autre individu de caractéristiques similaires. Ainsi, l'évolution du $R R$ va être instantanée, et uniquement conditionnée par le niveau de réduction du risque et les caractéristiques $p$.

En revanche, le $R R$ d'un individu exposé à un niveau plus faible de pollution atmosphérique ne va pas - en l'état actuel des connaissances épidémiologiques - dépendre fortement des caractéristiques individuelles $p$, mais dépendra par contre de l'historique de son exposition et de la façon dont son organisme va progressivement éliminer les facteurs de risques supplémentaires. L'évolution du $R R$ ne sera pas instantanée et la forme fonctionnelle $g$ retenue est présentée dans la section 3 .

\subsubsection{Risque relatif et mortalité}

Bien que l'analyse puisse s'appliquer tant à la morbidité qu'à la mortalité, nous nous restreignons ici aux seuls effets mortels, si bien que le $R R$ va uniquement affecter les taux de mortalité.

Notons $D_{p, 0}$ le taux de mortalité qui prévaut dans une population caractérisée par $p$, avant la mise en place de la politique de prévention. Nous distinguons la part relative aux causes de décès affectées par la politique, $D_{p, 0}^{A}$ et la part indépendante de cette politique, $D_{p, 0}^{I}$ :

$$
D_{p, 0}=D_{p, 0}^{A}+D_{p, 0}^{I}
$$

Pour illustrer ceci, les décès liés aux accidents de la route ne sont pas influencés par le niveau de la pollution atmosphérique, de même que le nombre 
de décès de type cardio-vasculaire n'est pas affecté par une amélioration des infrastructures routières. ${ }^{4}$

Une fois la politique de prévention mise en place, la part indépendante du taux de mortalité reste inchangée alors que celle affectée par la politique de prévention évolue en fonction de $R R_{p, t}$, si bien que le taux de mortalité total $t$ années après la mise en oeuvre de la politique, évolue comme :

$$
D_{p, t}=\frac{R R_{p, t}}{R R_{p, 0}} D_{p, 0}^{A}+D_{p, 0}^{I}
$$

\subsection{Evaluation sanitaire de la mortalité}

En règle générale, les études portant sur les bénéfices attendus d'une politique de prévention ne tiennent pas compte des éventuels effets de latence, et considèrent simplement l'effet d'une baisse de la mortalité au sein de la population bénéficiaire. Ce faisant, elles établissent un nombre de décès évités - ou éventuellement un nombre d'années de vie gagnées - qui correspond au bénéfice attendu lors de la première année de la politique de prévention.

Lorsque des effets de long terme existent, l'effet de latence invalide cette façon de procéder pour deux raisons. D'une part, une politique de prévention menée pendant une année n'aura, durant cette année, que des effets partiels liés à la décroissance progressive des $R R$, et d'autre part, les effets de cette politique se prolongeront dans le futur.

Il convient donc de prendre en compte cette dimension temporelle par une méthodologie adaptée afin d'éviter une difficulté propre à l'étude de la mortalité. En effet, puisqu'un décès évité à une date donnée surviendra inéluctablement à une date ultérieure, une approche inadaptée pourrait conclure que les bénéfices d'une politique de prévention sont transitoires et s'avèrent nuls à long terme. Une telle conclusion surviendrait par exemple si l'occurrence des décès observés au cours du temps au sein d'une population bénéficiant de cette politique était recensée. Nous proposons de désamorcer ce piège potentiel pour les deux approches évoquées, en termes d'années de vie gagnées et de décès prématurés évités. 


\subsubsection{A pproche en terme d'années de vie gagnées}

Les bénéfices d'une politique de prévention nécessitent de comptabiliser des années de vie gagnées, ce que nous réalisons par le suivi d'une cohorte. Son état (stationnaire) initial est défini par les taux de mortalité observés avant la mise en oeuvre de la politique de prévention, et nous retenons l'âge $x$ des individus comme unique caractéristique $p$ de la population.

- Le nombre de personnes d'âge $x$ vivant à la date $0, N_{0}(x)$, est égal à $N_{0}(x)=\Pi_{y=0}^{y=x-1}\left(1-D_{0}(y)\right) N$, c'est à dire au produit de tous les taux de survie conditionnels avant l'âge $x$ multiplié par le nombre annuel de naissance $N$, supposé constant dans le temps.

- Le nombre de personnes d'âge $x$ vivant à la date $t$ est calculé à partir de celui des personnes d'âge $x-1$ vivant à $t-1$ selon le taux de survie à la date $t: \forall x \geq 1, \forall t \geq 1, N_{t}(x)=\left(1-D_{t}(x-1)\right) N_{t-1}(x-1)$, avec $D_{t}($. donné par l'équation 3 .

- Le nombre total d'années de vie vécues à la date $t$ est: $V_{t}=\Sigma_{x} N_{t}(x)$.

Le nombre d'années de vie gagnées l'année $t$ est $\left(V_{t}-V_{0}\right)$, que l'on notera :

$$
N A S(\tau, \Delta, t)
$$

En l'absence de politique de prévention, $V_{t}=V_{0} \forall t$, et le nombre d'années de vie gagnées est nul. Lorsqu'une politique est mise en place, le bénéfice total actualisé est égal à :

$$
B_{a}(\delta, \tau, \Delta)=\sum_{t=0}^{\infty} \frac{1}{(1+\delta)^{t}} N A S(\tau, \Delta, t)
$$

où $\delta$ correspond au taux d'actualisation annuel. L'actualisation reflète ici un taux de préférence temporel portant sur des années de vie gagnées à différentes dates (voir Viscusi et al., 1997 ou Frederik et al., 2002) et ne fait pas intervenir de composantes économiques de type effet de richesse ou coût d'opportunité. 
Afin de faciliter la comparaison des bénéfices résultant de différentes politiques de prévention, nous calculons le bénéfice annuel actualisé équivalent, défini formellement comme la valeur $B A_{a}$ telle que :

$$
\sum_{t=0}^{\Delta-1}\left(\frac{1}{(1+\delta)^{t}} B A_{a}\right)=B_{a}(\delta, \tau, \Delta)
$$

c'est à dire :

$$
B A_{a}(\delta, \tau, \Delta)=\left(\frac{1-\frac{1}{(1+\delta)}}{1-\frac{1}{(1+\delta)^{\Delta}}}\right) B_{a}(\delta, \tau, \Delta)
$$

\subsubsection{A pproche en termes de décès prématurés évités}

L'élaboration d'une analyse temporelle correcte en terme de décès évités nécessite de revenir sur le fondement conceptuel de la VED. Les approches établies sur les préférences individuelles, qu'elles soient observées sur les marchés (travail, logement, biens de protection) ou déclarées (méthode d'évaluation contingente) mettent en relation une variation de la probabilité de décès (à un âge donné $x$ ) et un consentement à payer correspondant. ${ }^{5}$ Par conséquent, la VED à l'âge $x$ représente une valorisation de la réduction de la mortalité à l'âge $x$.

Lorsqu'on s'intéresse aux effets d'une politique de prévention sur la mortalité, $\left[D_{0}(x)-D_{t}(x)\right]$ mesure la réduction du taux de mortalité de la population d'âge $x$ à la date $t$. A la date $t$, le nombre de décès prématurés évités à l'âge $x$ est $N_{t}(x)\left[D_{0}(x)-D_{t}(x)\right]$, et le CAP total de la population pour cette réduction de la mortalité à l'âge $x$, est égal à :

$$
N_{t}(x)\left[D_{0}(x)-D_{t}(x)\right] \operatorname{VED}(x)
$$

où $V E D(x)$ est la valeur d'évitement d'un décès d'âge $x$.

Le bénéfice total actualisé est alors égal à :

$$
B_{v}(\delta, \tau, \Delta)=\sum_{t=0}^{\infty} \frac{1}{(1+\delta)^{t}}\left(\sum_{x=0}^{\infty} N_{t}(x)\left[D_{0}(x)-D_{t}(x)\right] V E D(x)\right)
$$

L'actualisation est ici sur un taux de préférence temporel portant sur des décès évités à différentes dates, exempt de toute dimension économique, comme l'était celui portant sur des années de vie gagnées.

Le bénéfice annuel équivalent $\left(B A_{v}\right)$ est dérivé du bénéfice total de la même façon que dans l'équation 6 . 


\subsection{Valeur d'évitement d'un décès}

La détermination d'une valeur d'année de vie (VAV) à utiliser dans l'approche en termes d'années de vie gagnées n'est pas traitée ici. En effet, la suite de l'analyse reposant sur des comparaisons relatives, nous normalisons donc implicitement la VAV à 1 quel que soit l'âge auquel elle s'applique. ${ }^{6}$

Dans l'approche en termes de décès prématurés il est possible d'utiliser des VED dépendant de l'âge pour calculer le bénéfice total actualisé (voir l'équation 7). Afin d'étudier la sensibilité des résultats aux méthodes de valorisation, la forme de la dépendance entre âge et VED va être obtenue par deux approches différentes.

La première correspond aux résultats empiriques de Jones-Lee (Jones-Lee et al., 1985; ou Sommer et al., 1999 et UK DH, 1999 pour ses plus récents résultats), qui observe, sur la base de CAP déclarés, une relation entre âge et $\mathrm{VED}$ en U inversé. Elle est notée $V E D^{E}(x)$, et nous la représentons sur la figure 1 (en la normalisant de telle sorte que le maximum soit égal à 1). $B A_{v}^{E}(\delta, \tau, \Delta)$ représente les bénéfices annuels équivalents correspondants.

Il convient de noter que le CAP individuel pour réduire l'exposition à un risque de décès dépend fortement des caractéristiques de ce risque. En effet, pour un même niveau de réduction et une même population touchée, un risque familier, volontaire et contrôlable tel celui résultant d'un déplacement individuel est mieux accepté qu'un risque potentiellement catastrophique et de nature effrayante tel celui résultant d'une épidémie (cf. Slovic et al., 1980; ou UK DH, 1999). Il en résulte de façon logique que lorsqu'elles reposent sur des préférences individuelles, les valeurs monétaires reflètent ces disparités en termes d'acceptation du risque, ce qui autorise un niveau d'analyse plus fin encore, que nous n'aborderons pas.

La seconde résulte d'un modèle théorique simple d'espérance d'utilité sur une durée de vie incertaine (Maddison, 1997, ou Shepard et Zeckhauser, 1982). L'espérance d'utilité pour une personne d'âge $x$ est égale à :

$$
E(U)=\left[\sum_{t=0}^{\infty} \frac{1}{(1+\delta)^{t}}\left(\Pi_{y=0}^{y=t-1}\left(1-D_{0}(y+x)\right)\right) U\left(w_{x+t}\right)\right]
$$

où $w_{x+t}$ est la richesse à l'âge $x+t$. Nous supposons que $w_{x+t}=w$ est constant 
pour tout âge, ce qui conduit à :

$$
E(U)=\left[\sum_{t=0}^{\infty} \frac{1}{(1+\delta)^{t}}\left(\Pi_{y=0}^{y=t-1}\left(1-D_{0}(y+x)\right)\right)\right] U(w)
$$

La VED correspond au CAP marginal pour une réduction immédiate et d'une durée d'une année de la probabilité de décès. Par conséquent :

$$
V E D(x)=\frac{\left[\sum_{t=0}^{\infty} \frac{1}{(1+\delta)^{t}}\left(\Pi_{y=0}^{y=t-1}\left(1-D_{0}(y+x)\right)\right)\right] U(w)}{U^{\prime}(w)}
$$

Dans ce modèle théorique simple, les VED obtenues, notées $V E D^{T}(x)$, sont proportionnelles à l'espérance de vie actualisée, comme le représente le profil normalisé de la figure $1^{7}$ pour trois valeurs du taux d'actualisation. Elles sont, comme précédemment, normalisées à 1 par rapport au maximum qui est observé à l'âge $x=0$. Les bénéfices annuels équivalents correspondants au calcul de la VED pour chaque âge de décès sont notés $B A_{v}^{T}(\delta, \tau, \Delta)$.

Toutefois, l'utilisation d'une VED dépendant de l'âge n'est pas systématique. En effet, les données sanitaires ne permettent pas de connaître l'âge de décès avec précision lorsque la cause de mortalité est non spécifique, et les études fournissant des résultats d'enquête de façon aussi fine sont rares, si bien que la valeur utilisée correspond le plus souvent à l'âge moyen des décès évités.

Le bénéfice total actualisé de l'équation 7 est alors égal à :

$$
\bar{B}_{v}(\delta, \tau, \Delta)=\sum_{t=0}^{\infty} \frac{1}{(1+\delta)^{t}}\left[\left(\sum_{x=0}^{\infty} N_{t}(x)\left[D_{0}(x)-D_{t}(x)\right]\right) V E D(\bar{x}(t))\right]
$$

où $\bar{x}(t)$ désigne l'âge moyen des décès évité à la date $t$ et est égal à :

$$
\bar{x}(t)=\frac{\sum_{x=0}^{\infty} x N_{t}(x)\left[D_{0}(x)-D_{t}(x)\right]}{\sum_{x=0}^{\infty} N_{t}(x)\left[D_{0}(x)-D_{t}(x)\right]}
$$

Les deux approches précédentes relatives à la forme de la dépendance entre âge et VED sont utilisées pour déterminer la VED à l'âge moyen de décès, et on notera de façon logique $\overline{B A}_{v}^{E}(\delta, \tau, \Delta)$ et $\overline{B A}_{v}^{T}(\delta, \tau, \Delta)$ les bénéfices annuels équivalents correspondant respectivement à l'approche empirique et théorique. 
Nous disposons maintenant du cadre méthodologique permettant de prendre correctement en compte l'effet de latence lorsqu'une politique de prévention implique des effets de long terme, et d'étudier les effets des choix relatifs aux méthodes de valorisation de la mortalité. L'application suivante permet de quantifier l'impact de ces deux effets sur la désirabilité relative de deux politiques de prévention.

\section{A pplication à deux politiques de prévention}

Deux politiques de prévention sont étudiées, l'une diminuant de $50 \%$ le nombre de décès par accidents de la route, et l'autre d'un quart le niveau de pollution atmosphérique. $^{8}$

\subsection{Données}

L'Insee (1997) fournit les taux de mortalité par âge et par sexe toutes causes $\left(D_{0}(x), x=0, \ldots, 99\right)$, et Inserm (1994) les taux de mortalité pour les causes accidentelles. ${ }^{9}$ Les données sont extrapolées jusqu'à l'âge de 120 ans afin que la taille de la population tende vers zéro. La façon dont le $R R$ évolue lors de la mise en place (et éventuellement l'arrêt) de la politique de prévention doit être spécifiée.

Dans le cas de la diminution de la pollution atmosphérique, les données épidémiologiques sont insuffisantes pour caractériser la fonction $g$ de l'équation 1. Aussi, procédons-nous par analogie avec les observations effectuées sur le sevrage tabagique, se fondant sur la similitude des indicateurs sanitaires concernés (maladies cardio-vasculaires et respiratoires), vraisemblablement liée à des composants communs $\left(\mathrm{NO}_{2}\right.$, hydrocarbones polycycliques, substances carcinogènes,,...$){ }^{10}$

La fonction de risque utilisée pour traduire la fonction $g$ est similaire à celle obtenue par Lightwood et Glantz (1997), fondée sur une méta analyse de sept études sur le sevrage tabagique, et utilisée par ailleurs dans le domaine de la 
pollution atmosphérique (voir Leksell et Rabl, 2001) :

$$
R R_{p, t}=R R_{p}^{N E}+\left(R R_{p}^{E}-R R_{p}^{N E}\right) \times \exp \left(-\frac{t}{\tau}\right)
$$

où $R R_{p}^{E}$ et $R R_{p}^{N E}$ correspondent respectivement aux $R R$ d'une population caractérisée par $p$ soumise à une exposition de niveau $E$ et à une exposition réduite par la politique de prévention, de niveau $N E$. Le paramètre constant $\tau$ caractérise l'évolution du $R R$ en fonction du délai écoulé depuis la mise en place de la politique. Nous notons par convention une diminution instantanée du $R R$ par $\tau=0$.

Pour représenter l'évolution du $R R$ après l'arrêt de la politique, l'expression 8 est étendue au cas où la consommation tabagique reprend après une période de sevrage. L'évolution observée peut alors s'analyser mathématiquement en considérant le $R R$ comme étant proportionnel à un stock (cf. Leksell et Rabl, 2001). L'équation 8 reste valide pour $\Delta \geq t>0$, mais devient alors, pour $t>\Delta$ :

$$
R R_{p, t}=R R_{p}^{E}+\left(R R_{p, \Delta}-R R_{p}^{E}\right) \times \exp \left(-\frac{t-\Delta}{\tau}\right)
$$

Une analyse de sensibilité est menée sur le paramètre $\tau$ - afin de tenir compte de l'incertitude des études épidémiologiques - qui variera entre 0 et 10 dans les simulations. La méthodologie (en particulier le traitement différencié des effets de court et de long terme) et les autres paramètres retenus sont détaillés dans Chanel et al. (2001a) et ne sont que brièvement rappelés ci-dessous. L'indicateur de pollution choisi est l'indicateur particulaire PM10, ${ }^{11}$ l'exposition moyenne pondérée de la population française est de $23.5 \mu \mathrm{g} / \mathrm{m}^{3}$ (Filliger et al., 1999) et le $R R$ de mortalité retenu est 1.043 pour une variation de 10 $\mu \mathrm{g} / \mathrm{m}^{3}$ de PM10 (Künzli et al., 2000). ${ }^{12}$

Dans le cas de la diminution des accidents de la route, Inserm (1994) fournit les taux de mortalité pour les causes accidentelles. Les bénéfices étant instantanés, $\tau=0$ et les équations 8 et 9 se simplifient grandement.

Le taux d'actualisation $\delta$, qui représente en fait un taux de préférence temporel portant sur des années de vie gagnées ou des décès évités à différentes dates, variera entre $1 \%$ à $8 \%$ par an, s'appuyant sur la récente revue critique 
de Frederik et al. (2002) sur ce sujet, qui fait état de valeurs comprises entre $0 \%$ et $17 \%$.

Afin d'éviter le caractère subjectif associé au choix d'une $V E D$ et d'une $V A V$ particulières, les résultats sont normalisés, tout comme l'étaient les formes fonctionnelles retenues pour représenter la dépendance de la VED à l'âge présentées dans la figure $1 .^{13}$

\subsection{Comparaison des bénéfices en termes sanitaires}

Pour les deux politiques de prévention considérées (réduction d'un quart de l'exposition à la pollution atmosphérique et de moitié des décès sur les routes), le nombre annuel d'années de vie gagnées est calculé à partir d'une population hypothétique constituée d'une cohorte de 100'000 nouvelles naissances chaque année. La cohorte, initialement à l'état stationnaire, évolue en fonction des nouveaux taux de mortalité (voir équation 3). L'état stationnaire final de long terme dépendra du caractère transitoire $(\Delta=1)$ ou définitif $(\Delta=\infty)$ de la politique mise en oeuvre. ${ }^{14}$

Dans le premier cas, l'état stationnaire final correspond à l'état stationnaire initial, et les résultats de la politique de lutte contre la pollution atmosphérique dépendent assez fortement de la valeur de $\tau$ qui traduit la vitesse à laquelle les $R R$ vont décroître. Ainsi, si l'on suppose qu'elle est instantanée (autrement dit, que l'ensemble des effets sont des effets de court terme), les bénéfices vont croître très rapidement, puis décroître presqu'aussi rapidement. Plus $\tau$ est élevé, moins le nombre d'années de vie gagnées est important, et plus le nombre d'années pour l'atteindre puis pour revenir à 0 est élevé (voir figure 2). Pour la politique de prévention routière, la valeur de $\tau$ n'intervient pas puisque les effets sont instantanés. Le nombre d'années de vie gagnées est plus faible, mais le délai nécessaire pour retrouver l'état stationnaire initial est long.

Dans le cas d'une politique permanente, le nombre d'années de vie gagnées va croître de façon continue jusqu'à atteindre un maximum à l'état stationnaire final, après 50 ans pour la politique de lutte contre la pollution atmosphérique et 80 ans pour celle de prévention routière. Bien que la différence entre le nombre d'années de vie gagnées à l'état stationnaire soit relativement faible, 
l'aire entre les deux courbes les représentant est assez importante pour de faibles valeurs de $\tau$ (voir figure 3 ).

Cette analyse ne tient pas compte des âges auxquels ces années de vie sont gagnées, bien qu'ils diffèrent fortement comme l'indique la figure 4. Celle-ci représente la distribution des âges de décès associée à chacune des politiques, établie d'après Inserm (1994) pour les accidents de la route, et par différence entre les nombres de survivants des états stationnaires finals et initiaux pour les $R R$ retenus précédemment et $\Delta=\infty$. L'âge moyen calculé à partir de ces distributions est également mentionné : 39 ans pour les accidents de la route et 77 ans pour l'exposition à la pollution atmosphérique. L'analyse en termes monétaires permet maintenant d'introduire explicitement la dimension associée à l'âge de décès, de façon plus ou moins fine selon qu'elle s'applique à l'ensemble de la distribution ou à l'âge moyen de décès.

\subsection{Comparaison des bénéfices en termes monétaires}

La sensibilité aux choix méthodologiques des bénéfices économiques des deux politiques est étudiée. Les valeurs de référence suivantes, notées Ref dans la suite, sont retenues :

- un taux d'actualisation de $4 \%(\delta=4 \%)$,

- une durée de 1 an pour la politique de prévention $(\Delta=1)$,

- l'absence de prise en compte de l'effet de latence lors d'une réduction de la pollution de l'air $(\tau=0)$.

La sensibilité aux différents choix - paramétriques et méthodologiques - de l'évaluation de chacune des deux politiques est d'abord étudiée, avant d'effectuer une comparaison des deux politiques.

\subsubsection{Sensibilité des évaluations}

Les tableaux 1 et 2 présentent, pour chaque politique, l'effet des paramètres sur l'évaluation. Le ratio $\frac{B A(\delta, \tau, \Delta)}{B A(R e f)}$ est donc calculé pour différentes valeurs des 
paramètres $\delta, \Delta$ et $\tau$ pour la pollution atmosphérique et vaut 1 pour la situation de référence (encadrée dans le tableau 1). Les cinq méthodes de valorisation monétaire présentées précédemment sont étudiées. L'impact du paramètre de durée de la politique $\Delta$ s'avérant négligeable sur le ratio ${ }^{15}$ (moins de $1 \%$ ), seuls les résultats correspondants à une politique d'une durée $\Delta=1$ an sont présentés.

L'évaluation économique d'une politique de prévention routière est sensible au choix du taux d'actualisation pour l'approche en termes d'année de vie (seconde colonne du tableau 1). Ceci s'explique par le fait que les gains en années de vie s'étalent dans le temps (voir figure 2), même si la politique ne dure qu'une seule année. Par conséquent, le taux d'actualisation réduit logiquement la valeur économique de la politique, et de façon assez importante puisque les bénéfices sont pratiquement divisés par 3 lorsque l'on passe d'un taux de $1 \%$ à un taux de $8 \%$.

Tableau 1 Sensibilité des résultats (prévention routière, $\Delta=1$ an)

\begin{tabular}{cccccc}
\hline Ratios & $\frac{B A_{\mathrm{a}}(\delta, \tau, \Delta)}{B A_{\mathrm{a}}(\text { Ref })}$ & $\frac{B A_{\mathrm{V}}^{\mathrm{E}}(\delta, \tau, \Delta)}{B A_{\mathrm{V}}^{\mathrm{E}}(\text { Ref })}$ & $\frac{\overline{B A}_{\mathrm{v}}^{\mathrm{E}}(\delta, \tau, \Delta)}{\overline{B A}_{\mathrm{V}}^{\mathrm{E}}(\text { Ref })}$ & $\frac{B A_{\mathrm{v}}^{\top}(\delta, \tau, \Delta)}{B A_{\mathrm{V}}^{\top}(\operatorname{Ref})}$ & $\frac{\overline{B A}_{\mathrm{V}}^{\top}(\delta, \tau, \Delta)}{\overline{B A}_{\mathrm{V}}^{\top}(\text { Ref })}$ \\
\hline$\delta=1 \%$ & 1,67 & 1,00 & 1,00 & 0,79 & 0,77 \\
\cline { 2 - 7 }$\delta=4 \%$ & 1,00 & 1,00 & 1,00 & 1,00 & 1,00 \\
\cline { 2 - 7 }$\delta=8 \%$ & 0,64 & 1,00 & 1,00 & 1,09 & 1,08 \\
\hline
\end{tabular}

Pour l'approche en termes de décès évités, les bénéfices sont obtenus sans délai et il est donc logique que l'évaluation économique soit indépendante du taux d'actualisation pour les valeurs de la vie humaine fondées sur les modèles empiriques (troisième et quatrième colonnes du tableau 1), qui ne font pas intervenir $\delta$.

Enfin, lorsque la valeur de la vie humaine est fondée sur un modèle théorique (cinquième et sixième colonne du tableau 1), l'augmentation du taux d'actualisation a pour effet de réduire l'écart relatif entre les VED relatives à des âges élevés et les VED relatives à des âges moindres (voir figure 1). Ceci explique que le bénéfice monétaire augmente avec le taux d'actualisation dans les deux cas.

Dans le cas de la politique de lutte contre la pollution de l'air, la sensibilité 
des résultats au taux d'actualisation est comparable à celle observée pour la politique de prévention routière selon les méthodes de valorisation utilisées (tableau 2). Notons toutefois que son amplitude est réduite pour l'approche en termes d'année de vie (passage de 1.24 à 0.81) et augmentée pour l'approche en terme de VED fondée sur un modèle théorique (passage de 0.6 à 1.26). Ceci s'explique par le fait que la réduction de la mortalité va concerner des âges plus avancés que ceux associés à la politique de prévention routière.

Tableau 2 Sensibilité des résultats (prévention de la pollution de l'air, $\Delta=1$ an)

\begin{tabular}{|c|c|c|c|c|c|c|c|c|c|c|c|c|c|c|c|c|c|c|c|c|}
\hline \multirow{2}{*}{$\frac{\text { Ratios }}{\tau}$} & \multicolumn{4}{|c|}{$\frac{B A_{\mathrm{a}}(\delta, \tau, \Delta)}{B A_{\mathrm{a}}(\operatorname{Ref})}$} & \multicolumn{4}{|c|}{$\frac{B A_{\mathrm{V}}^{\mathrm{E}}(\delta, \tau, \Delta)}{B A_{\mathrm{V}}^{\mathrm{E}}(R e f)}$} & \multicolumn{4}{|c|}{$\frac{\overline{B A}_{\mathrm{V}}^{\mathrm{E}}(\delta, \tau, \Delta)}{\overline{B A}_{\mathrm{V}}^{\mathrm{E}}(R e f)}$} & \multicolumn{4}{|c|}{$\frac{B A_{\mathrm{V}}^{\top}(\delta, \tau, \Delta)}{B A_{\mathrm{\vee}}^{\top}(\operatorname{Ref})}$} & \multicolumn{4}{|c|}{$\frac{\overline{B A}_{\mathrm{v}}^{\top}(\delta, \tau, \Delta)}{\overline{B A}_{\mathrm{V}}^{\top}(\operatorname{Ref})}$} \\
\hline & 0 & 1 & 5 & 10 & 0 & 1 & 5 & 10 & 0 & 1 & 5 & 10 & 0 & 1 & 5 & 10 & 0 & 1 & 5 & 10 \\
\hline$\delta=1 \%$ & 1,24 & 1,24 & 1,20 & 1,15 & 1,00 & 1,00 & 0,96 & 0,93 & 1,00 & 1,00 & 0,96 & 0,93 & 0,60 & 0,60 & 0,58 & 0,56 & 0,59 & 0,58 & 0,57 & 0,54 \\
\hline$\delta=4 \%$ & 1,00 & 0,98 & 0,87 & 0,77 & 1,00 & 0,98 & 0,87 & 0,77 & 1,00 & 0,98 & 0,87 & 0,77 & $\square, 00$ & 0,98 & 0,87 & 0,77 & 1,00 & 0,98 & 0,87 & 0,77 \\
\hline$\delta=8 \%$ & 0,81 & 0,78 & 0,63 & 0,52 & 1,00 & 0,96 & 0,78 & 0,64 & 1,00 & 0,96 & 0,78 & 0,64 & 1,26 & 1,21 & 0,99 & 0,81 & 1,27 & 1,22 & 0,99 & 0,82 \\
\hline
\end{tabular}

Concernant l'effet de la prise en compte correcte de l'effet de latence dans l'évaluation économique ( $\tau>0$ vs. $\tau=0$ ), on observe un effet négatif sur l'évaluation des bénéfices. Si l'effet du délai de latence est similaire quelle que soit l'approche de valorisation retenue, ${ }^{16}$ il est renforcé par le niveau du taux d'actualisation. En effet, passer de $\tau=0$ à $\tau=10$ se traduit pas une réduction de $7 \%$ quand $\delta=1 \%$, mais de $35 \%$ quand $\delta=8 \%$.

Les tableaux 1 et 2 ne peuvent être comparés l'un à l'autre en termes monétaires puisque la standardisation effectuée par le ratio est propre à chaque politique. Ils peuvent cependant être comparés en termes relatifs.

\subsubsection{Sensibilité de la comparaison}

Cette section étudie la façon dont la prise en compte du temps de latence, les choix méthodologiques et les valeurs des paramètres peuvent influencer la comparaison des deux politiques par rapport à la situation de référence dans laquelle les bénéfices des deux politiques sont jugés égaux. L’approche monétaire de référence retenue est l'approche empirique en termes de décès évités ramenés à un âge moyen $\left(\overline{B A}_{v}^{E}\right)$, qui nous semble représentative des 
évaluations économiques proposées couramment dans la littérature.

Nous étudions donc le rapport des ratios $\frac{B A(\delta, \tau, \Delta)}{\overline{B A}_{\mathrm{v}}^{\mathrm{E}}(\delta=4 \%, \tau=0, \Delta=1)}$, celui de la politique de Prévention Routière $(\mathrm{PR})$ étant au numérateur et celui de la politique de réduction de la Pollution Atmosphérique (PA) au dénominateur. L'attention doit être attirée sur l'interprétation des résultats de ce ratio. Un ratio supérieur à 1 signifie que, par rapport à la situation de référence Ref et $\overline{B A}_{v}^{E}$ pour laquelle le ratio vaut 1, l'intérêt économique de la politique de prévention routière sera renforcée. Des paramètres conduisant à une valeur inférieure à 1 seront au contraire favorables à la politique de réduction de la pollution de l'air. La valeur du ratio ne constitue donc qu'une mesure quantitative de l'évolution comparative des bénéfices économiques des deux politiques en fonction des valeurs des paramètres.

Tableau 3 Sensibilité de la comparaison des politiques $(\Delta=1$ an)

\begin{tabular}{|c|c|c|c|c|c|c|c|c|c|c|c|c|c|c|c|c|c|c|c|c|}
\hline \multirow{2}{*}{$\frac{\text { Ratios }}{\tau}$} & \multicolumn{4}{|c|}{$\frac{B A_{\mathrm{a}} / \overline{B A}_{\mathrm{V}}^{\mathrm{E}}(R e f)^{\mathrm{P} \mathrm{R}}}{B A_{\mathrm{a}} / \overline{B A}_{\mathrm{V}}^{\mathrm{E}}(R e f)^{\mathrm{PA}}}$} & \multicolumn{4}{|c|}{$\frac{B A_{\mathrm{V}}^{\mathrm{E}} / \overline{B A}_{\mathrm{V}}^{\mathrm{E}}(R e f)^{\mathrm{P} \mathrm{R}}}{B A_{\mathrm{V}}^{\mathrm{E}} / \overline{B A}_{\mathrm{V}}^{\mathrm{E}}(R e f)^{\mathrm{PA}}}$} & \multicolumn{4}{|c|}{$\frac{\overline{B A}_{\mathrm{V}}^{\mathrm{E}} / \overline{B A}_{\mathrm{V}}^{\mathrm{E}}(R e f)^{\mathrm{P} \mathrm{R}}}{\overline{B A}_{\mathrm{V}}^{\mathrm{E}} / \overline{B A}_{\mathrm{V}}^{\mathrm{E}}(R e f)^{\mathrm{PA}}}$} & \multicolumn{4}{|c|}{$\frac{B A_{\mathrm{V}}^{\mathrm{T}} / \overline{B A}_{\mathrm{V}}^{\mathrm{E}}(R e f)^{\mathrm{P} \mathrm{R}}}{B A_{\mathrm{V}}^{\mathrm{T}} / \overline{B A}_{\mathrm{V}}^{\mathrm{E}}(R e f)^{\mathrm{PA}}}$} & \multicolumn{4}{|c|}{$\frac{\overline{B A}_{\mathrm{V}}^{\top} / \overline{B A}_{\mathrm{V}}^{\mathrm{E}}(R e f)^{\mathrm{P} \mathrm{R}}}{\overline{B A}_{\mathrm{V}}^{\top} / \overline{B A}_{\mathrm{V}}^{\mathrm{E}}(R e f)^{\mathrm{P} \mathrm{A}}}$} \\
\hline & 0 & 1 & 5 & 10 & 0 & 1 & 5 & 10 & 0 & 1 & 5 & 10 & 0 & 1 & 5 & 10 & 0 & 1 & 5 & 10 \\
\hline$\delta=1 \%$ & 1,41 & 1,42 & 1,47 & 1,52 & 0,68 & 0,68 & 0,71 & 0,73 & 1,00 & 1,00 & 1,04 & 1,08 & 0,88 & 0,89 & 0,91 & 0,95 & 0,90 & 0,90 & 0,93 & 0,97 \\
\hline$\delta=4 \%$ & 1,05 & 1,07 & 1,20 & 1,36 & 0,68 & 0,69 & 0,78 & 0,88 & 1,00 & 1,02 & 1,15 & 1,30 & 0,67 & 0,69 & 0,77 & 0,88 & 0,69 & 0,70 & 0,78 & 0,89 \\
\hline$\delta=8 \%$ & 0,83 & 0,84 & 1,07 & 1,29 & 0,68 & 0,70 & 0,87 & 1,05 & 1,00 & 1,04 & 1,27 & 1,55 & 0,59 & 0,61 & 0,75 & 0,91 & 0,58 & 0,60 & 0,74 & 0,90 \\
\hline
\end{tabular}

L'observation du tableau 3 conduit aux résultats suivants (la situation de référence est encadrée).

- Quelle que soit la méthode de valorisation retenue, plus l'effet de latence $(\tau)$ de la politique de prévention de la pollution de l'air est élevé, plus le ratio est élevé. Ceci est conforme à l'intuition puisque les gains sanitaires seront obtenus plus tardivement (et seront de plus moins élevés dans le cas d'une politique transitoire).

- L'effet de latence se conjugue par ailleurs à celui de l'actualisation. La colonne correspondant à $\overline{B A}_{v}^{E}$ constitue une bonne indication de la sensibilité des résultats aux effets joints de ces deux paramètres : le pourcentage d'augmentation de ce ratio par rapport à la situation de référence 
est négligeable pour $\tau=1$ et $\delta=1 \%$, de près de $55 \%$ pour $\tau=10$ et $\delta=8 \%$, et de $15 \%$ pour $\tau=5$ et $\delta=4 \%$.

- L'actualisation joue également lors de la monétarisation (à l'exception des valeurs de VED empiriques). Une augmentation du taux d'actualisation réduit ainsi l'intérêt relatif de la politique de prévention routière, comme le montrent les résultats des deuxième, cinquième et sixième colonnes. En effet, dans le modèle théorique de la VED, un augmentation du taux d'actualisation réduit la différence relative entre les VED à des âges moyens (utilisées pour la prévention routière) et à des âges élevés (utilisées pour la prévention de la pollution de l'air). Cette réduction relative opère également pour l'approche en termes d'années de vie gagnées puisqu'un décès évité sur la route correspond à environ 4 fois plus d'années de vie qu'un décès attribuable à la pollution de l'air (41 ans contre 10 ans). La mécanique de l'actualisation implique que 10 ans actualisés à $\delta=4 \%$ représentent $40 \%$ de 41 ans, et même $60 \%$ lorsque $\delta=8 \%$.

- L'approche en termes d'années de vie gagnées (seconde colonne) se distingue des quatre approches fondées sur une VED puisqu'elle renforce de manière importante l'intérêt relatif de la politique de prévention routière quand elle est appliquée avec un taux d'actualisation faible ou modéré. En effet, elle valorise d'autant plus fortement les décès évités d'âge faible que le taux d'actualisation est faible, alors que, quelle que soit la méthode retenue, la VED à l'âge de 77 ans vaut au moins la moitié de la VED à l'âge de 39 ans quel que soit $\delta$ (voir figure 1).

- Comparée aux trois autres approches en termes de VED, l'approche de référence (VED empirique calculée à l'âge moyen) favorise la politique de prévention routière car l'âge moyen des décès par accidents de la route (39 ans) est proche de la valeur maximale. On constate que la simplification, fréquente dans l'analyse économique qui consiste à utiliser un âge moyen, possède des conséquences importantes dans les cas où le profil de la VED n'est pas décroissant avec l'âge. ${ }^{17}$ Par contre, pour 
le profil de VED donné par le modèle théorique, les résultats sont peu sensibles au choix de l'âge moyen en lieu de l'âge réel des décès (cinquième et sixième colonnes).

Ainsi, les choix méthodologiques revêtent une importance quantitative importante dans le calcul et la comparaison de politiques de prévention, puisqu'un ratio de 0,66 par exemple signifie que les bénéfices d'une politique de prévention routière sont réduits d'un tiers comparativement à ceux d'une politique de lutte contre la pollution de l'air.

\section{Conclusion}

Cet article prend en compte l'effet de latence par une méthodologie adaptée et compare plusieurs méthodes de valorisation économique de la mortalité. Les simulations réalisées montrent que la prise en compte de l'effet de latence modifie substantiellement les bénéfices attendus d'une politique de prévention, et que le choix de la méthode de monétarisation importe également.

En effet, choisir une approche en termes de VAV ou en termes de VED modifie considérablement les résultats. D'un point de vue normatif, il semble que l'approche correcte soit une approche en termes de VED, fondée sur des résultats empiriques et tenant compte de l'âge réel de décès. Une telle approche prend en effet en compte les préférences réelles des agents économiques et mesure correctement les bénéfices. La comparaison des résultats de la troisième colonne du tableau 3 à ceux de la seconde (approche en termes d'années de vie gagnées) laisse apparaître un écart important entre les approches en termes de VAV et de VED.

Ceci confirme que la comparaison d'évaluations économiques en économie de l'environnement et en économie de la santé est délicate, les approches retenues dans ces deux domaines étant différentes. En économie de l'environnement, certains économistes jugent souhaitable de passer à une approche en terme d'années de vie pour éviter le problème de la définition d'une VED dépendant de l'âge. 
Bien que jugeant cette approche préférable, la Commission Boiteux (Boiteux et Baumstark, 2001) au Commissariat Général du Plan, a proposé de nouvelles VED à utiliser pour les études économiques de l'Administration française. Pour chiffrer les nuisances générées par les émissions des transports routiers, elle retient une VED associée à la pollution de l'air égale à 35\% de la VED associée à un accident dans le secteur des transports qui est de 1,5 millions d'euros. Cette valeur de $35 \%$ résulte d'une part de la prise en compte de l'espérance de vie actualisée, qui implique un coefficient de $56 \%$ pour $\delta=8 \%$, et d'autre part, de l'application d'un facteur rendant compte de la qualité de vie plus faible lors du décès. Les résultats de la troisième section indiquent que procéder ainsi favorise fortement une politique de prévention routière plutôt qu'une politique de réduction de la pollution atmosphérique. Faut-il alors considérer l'intérêt inégal qu'accorde actuellement le gouvernement à ces deux politiques comme le résultat d'une analyse économique cohérente de leurs bénéfices respectifs ? A moins qu'il ne reflète simplement les préoccupations de la majorité de la population envers les caractéristiques des risques sous-jacents. 


\section{Notes}

${ }^{1}$ L'annonce du plan Vieillissement et Solidarité suite à la canicule de l'été 2003 en constitue une illustration, bien qu'il ait vocation à couvrir des aspects beaucoup plus larges que la seule mortalité des personnes âgées.

${ }^{2}$ Rappelons qu'à la naissance, l'espérance de vie moyenne est de 77,8 ans environ, qu'elle est de 40,7 ans environ à l'âge de 39 ans et de 10,2 ans à l'âge de 77 ans (Insee, 1997).

${ }^{3}$ Le lecteur trouvera dans Chanel et al. (2001a) une étude des bénéfices associés à des politiques de réduction s'étalant sur diverses durées et portant sur plusieurs niveaux de réduction.

${ }^{4}$ D'aucuns feront toutefois remarquer qu'une diminution des ressources médicales consacrées au traitement d'un problème sanitaire peut théoriquement donner lieu à une réallocation des ressources susceptible de diminuer les taux de mortalité relatifs à d'autres causes de décès. Ce phénomène, de toute évidence marginal, dépasse le cadre de notre analyse.

${ }^{5}$ Nous laissons de côté les VED fondées sur les pertes de productions actualisées ou les décisions réglementaires, qui nous apparaissent marginales et très critiquables.

${ }^{6}$ Une méthode directe pour obtenir une VAV consiste à utiliser les résultats de l'expression de CAP individuels pour accroître d'un an son espérance de vie. Malheureusement, il existe très peu d'enquêtes de ce type (voir toutefois Johannesson et Johansson, 1997; ou Krupnick et al., 1999) et les résultats dépendent fortement de l'âge du répondant et de l'horizon auquel cette année de vie serait gagnée. Une méthode indirecte permet pour sa part de déduire une VAV à partir d'une VED en considérant que cette dernière est la somme actualisée d'années de même valeur (voir par exemple Viscusi et al., 1997; ou Leksell et Rabl, 2001).

${ }^{7}$ En choisissant une VED de référence pour un âge donné (généralement 40 ans), ce modèle permet de calculer des VED pour tous les âges.

${ }^{8} \mathrm{La}$ moitié environ de la pollution de l'air d'origine anthropique étant attribuée au trafic routier (Künzli et al., 2000), une diminution du quart du niveau de pollution atmosphérique peut être le résultat d'une diminution de moitié la part due au trafic routier. Ainsi, les deux politiques étudiées réduisent de moitié deux facteurs de risque de décès liés au trafic routier (celui associé à une exposition à la pollution et celui associé à un accident) et ne semblent pas totalement irréalistes.

${ }^{9} \mathrm{La}$ tendance récente à la diminution des accidents de la route n'est pas prise en compte.

${ }^{10}$ Notons au passage qu'une politique de prévention du tabagisme s'implémenterait donc de la même façon en utilisant les $R R$ de mortalité associés aux expositions tabagiques des fumeurs actifs et passifs.

${ }^{11}$ Particules en suspension d'un diamètre inférieur à $10 \mu \mathrm{m}$. 
${ }^{12}$ Les données épidémiologiques établissent que l'exposition à la pollution atmosphérique entraîne des effets sur la mortalité à court terme $\left({ }^{\mathrm{CT}} R^{\mathrm{E}}=0.006\right)$ et à long terme $\left({ }^{\mathrm{LT}} R^{\mathrm{E}}=\right.$ 0.037) parmi la population âgée de 30 ans et plus. Ceci a été pris en compte dans les calculs.

${ }^{13}$ Notons à ce propos que la forme de la dépendance entre la VED empirique et l'âge est issue de travaux de Jones-Lee et al. (1985) pour un risque associé aux accidents de la route. La pertinence de son utilisation pour valoriser des décès attribués à la pollution de l'air mérite une justification compte-tenu de l'influence des caractéristiques du risque sous-jacent sur les VED dérivées de préférences individuelles. A notre connaissance, seuls Chanel et al. (2001b) proposent une VED dépendant de l'âge dans le cadre d'une évaluation contingente portant explicitiment sur un risque d'exposition à la pollution de l'air. Ils obtiennent une forme fonctionnelle quadratique normalisée très proche de celle de Jones-Lee et al. (1985) avec un maximum à l'âge de 43 ans au lieu de 48 ans. Ainsi, afin d'éviter un degré de complexité supplémentaire, la forme fonctionnelle de la figure 1 est retenue pour monétariser les décès évités par les deux politiques.

${ }^{14}$ Les résultats ne sont donnés que pour les deux cas polaires $\Delta=1$ et $\Delta=\infty$ car il s'avère que ceux des politiques intermédiaires sont comparables.

${ }^{15} \mathrm{Ce}$ n'est bien évidemment pas le cas en termes absolus.

${ }^{16}$ On constate par exemple que la réduction des bénéfices est de $23 \%$ lorsque l'on passe de $\tau=0$ à $\tau=10$ (pour $\delta=4 \%$ ), quelle que soit l'approche.

${ }^{17}$ Notons que cette pratique courante possède une dimension éthique puisqu'une VED similaire pour chaque âge ne prête pas à la polémique. 


\section{Références}

Bleichrodt, H. (1995). QALYs and HYEs: Under what Conditions are they Equivalent. J ournal of Health Economics, 14, 17-37.

Boiteux, M. et L. Baumstark (2001). Transports : choix des investissements et coûts des nuisances. Paris, La Documentation Française.

Chanel, O., P. Scapecchi et J.-C. Vergnaud (2001a). Long term health effects and economic valuation of public policies: an application to air pollution in France. DT Greqam 01B03, http://www.vcharite.univ-mrs.fr/GREQAM.

Chanel, O., E. Faugère, G. Geniaux, R, Kast, S. Luchini, C. Protopopescu et P. Scapecchi (2001b). Approche économique et socio-anthropologique de la pollution atmosphérique : application au département des Bouches-du-Rhône. Etude pour le Ministère de l'Aménagement du Territoire et de l'Environnement, Primequal 36/98.

Filliger, P., V. Puybonnieux-Texier et J. Schneider (1999). Health Costs due to Road Traffic-related Air Pollution, an Impact Assessment Project of Austria, France and Switzerland, PM10 Population Exposure. WHO Technical Report TEH05.

Frederick, S., G. Loewenstein et T. O'Donoghue (2002). Time Discounting and Time Preference: A Critical Review. J ournal of Economic Literature, 40:2, 351-401.

Hammitt, J. (2003). Valuing Health: Qualited-adjusted Life Years or Willingness to Pay? Risk in perspective, Harvard Center for Risk Analysis, 11, $\mathrm{n}^{\circ} 1$.

INSEE (1997). La Situation Démographique en 1995. Démographie-Société 65-66.

INSERM (1994). Statistiques des Causes Médicales de Décès. INSERM SC8-SC25.

Johannesson, M. et P.O. Johansson (1996). To Be or not to Be, that is the Question: An Empirical Study of the WTP for an Increased Life Expectancy at an Advanced Age. J ournal of Risk and Uncertainty, 13, 163-174.

Johannesson, M. et P.O. Johansson (1997). Quality of Life and the WTP for an Increased Life Expectancy at an Advanced Age. J ournal of Public 
Economics, 65, 219-228.

Jones-Lee, M, M. Hammerton et P.R. Philips (1985). The Value of Safety: Results of a National Sample Survey. The Economic J ournal, 95, 49-72.

Kaplan, E.H. et M.H. Merson (2002). Allocating HIV-prevention resources: balancing efficiency and equity. A merican J ournal of Public Health, 92(12), 1905-1907.

Krupnick, A.J., A. Alberini, M. Cropper, N. Simon with K. Itaoka et M. Akai (1999). Mortality Risk Valuation for Environmental Policy, Discussion paper Resources for the Future 99-47.

Krupnick, A., A. Alberini, M. Cropper, N. Simon, B. O’Brien, R. Goeree et M. Heintzelman (2002). Age, Health and the Willingness to Pay for Mortality Risk Reductions: A Contingent Valuation Survey of Ontario Residents. J ournal of Risk and Uncertainty 24, 161-186.

Künzli, N., R. Kaiser, S. Medina, M. Studnicka, O. Chanel, M. Herry, F. Horak, V. Puybonnieux-Texier, P. Quénel, J., Schneider, R. Seethaler, J.-C. Vergnaud et H. Sommer (2000). Public-health Impact of Outdoor and Trafficrelated Air Pollution: a European Assessment. Lancet 356, 795-801.

Leksell, I. et A. Rabl (2001). Air Pollution and Mortality: Quantification and Valuation of Years of Life Lost. Risk Analysis 21(5), 843-857.

Lightwood, J.M. et S.A. Glantz (1997). Short-term Economic and Health Benefits of Smoking Cessation. Circulation 96, 1089-1096.

Lindholm, L., M. Rosen et M. Emmelin (1996). An Epidemiological Approach Towards Measuring the Trade-off Between Equity and Efficiency in Health Policy. Health Policy 35(3), 205-216.

Loomes, G. et L. McKenzie (1989). The use of Qaly's in health care decision making. Social Science and Medicine 28, 299-308.

Maddison D. (1997). Valuing Health Impacts form Air Pollution. Mimeographed, CSERGE, University College London and University of East Anglia.

Moatti, J.P. (1997). Priorités de santé publique : Les dangers d'une dérive utilitariste. Haut Comité de la santé publique, Actualité et dossier en santé publique $\mathrm{n}^{\circ} 17$.

Pliskin, J., D. Shepard et M. Weinstein (1980). Utility Functions for Life 
Years and Health Status. Operations Research 28, 206-224.

Regens, J.L. (1991). Measuring Environmental Benefits with Contingent Markets. Public Administration Review 51(2), 345-352.

Shepard D.S. et R.J. Zeckhauser (1982). Life-Cycle Consumption and Willingness to Pay for Increased Survival. dans Jones-Lee M. (ed.), The Value of Life and Safety, North-Holland, New-York.

Slovic P., B. Fischhoff et S. Liechtenstein (1980). Facts and fears: understanding percieved risk. dans Schwing R. et Albers W.A. (ed.), Social Risk Assessment, New-York, Plenum.

Sommer, H., R. Seethaler, O. Chanel, M. Herry, S. Masson, et J.-C. Vergnaud (1999). Health Costs due to Road Traffic-related Air pollution, an Impact Assessment Project of Austria, France and Switzerland, Economic Valuation. WHO Technical Report TEH07.

Ubel, P.A., J. Baron, B. Nash et D.A. Asch (2000). Are Preferences for Equity over Efficiency in Health Care Allocation "All or Nothing"?. Medical Care, 38(4), 366-373.

UK DH (1999). Economic Appraisal of the Health Effects of Air Pollution, Ad-Hoc Group on the Economic Appraisal of the Health Effects of Air Pollution. London: The Stationary Office.

Viscusi K., J. Hakes and A. Carlin, (1997). Measure of Mortality Risks. The J ournal of Risk and Uncertainty, 14(3), 213-233. 


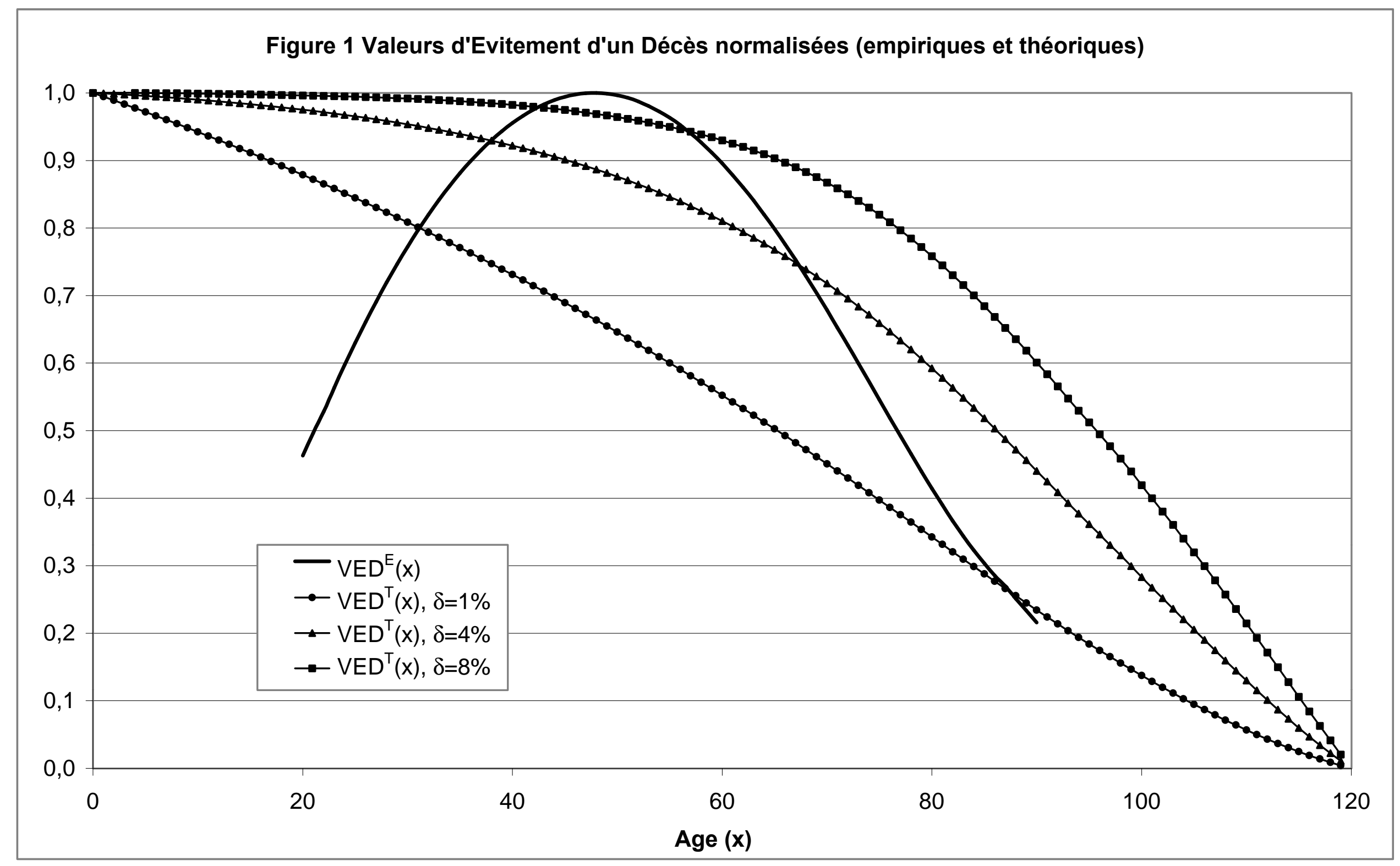




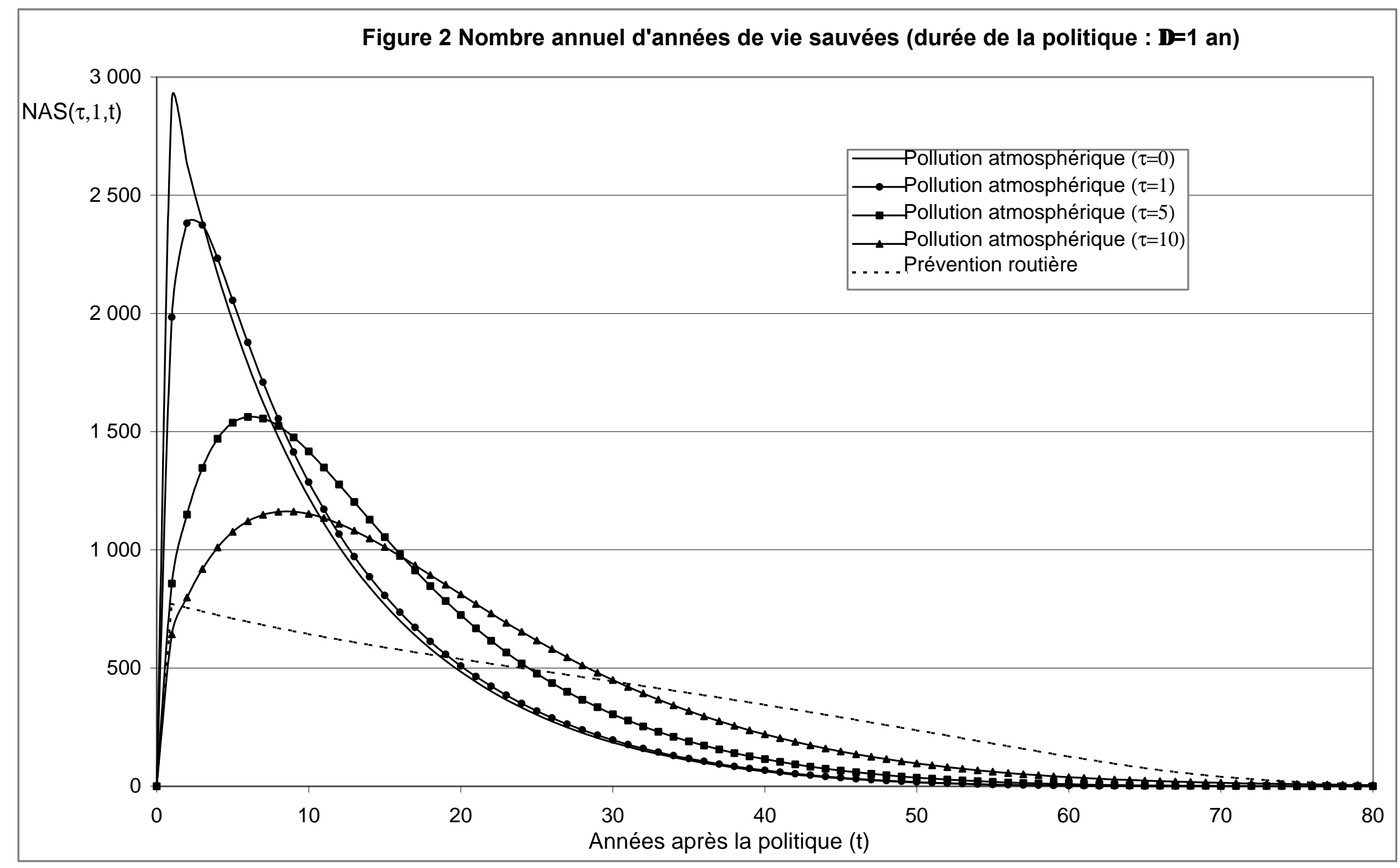




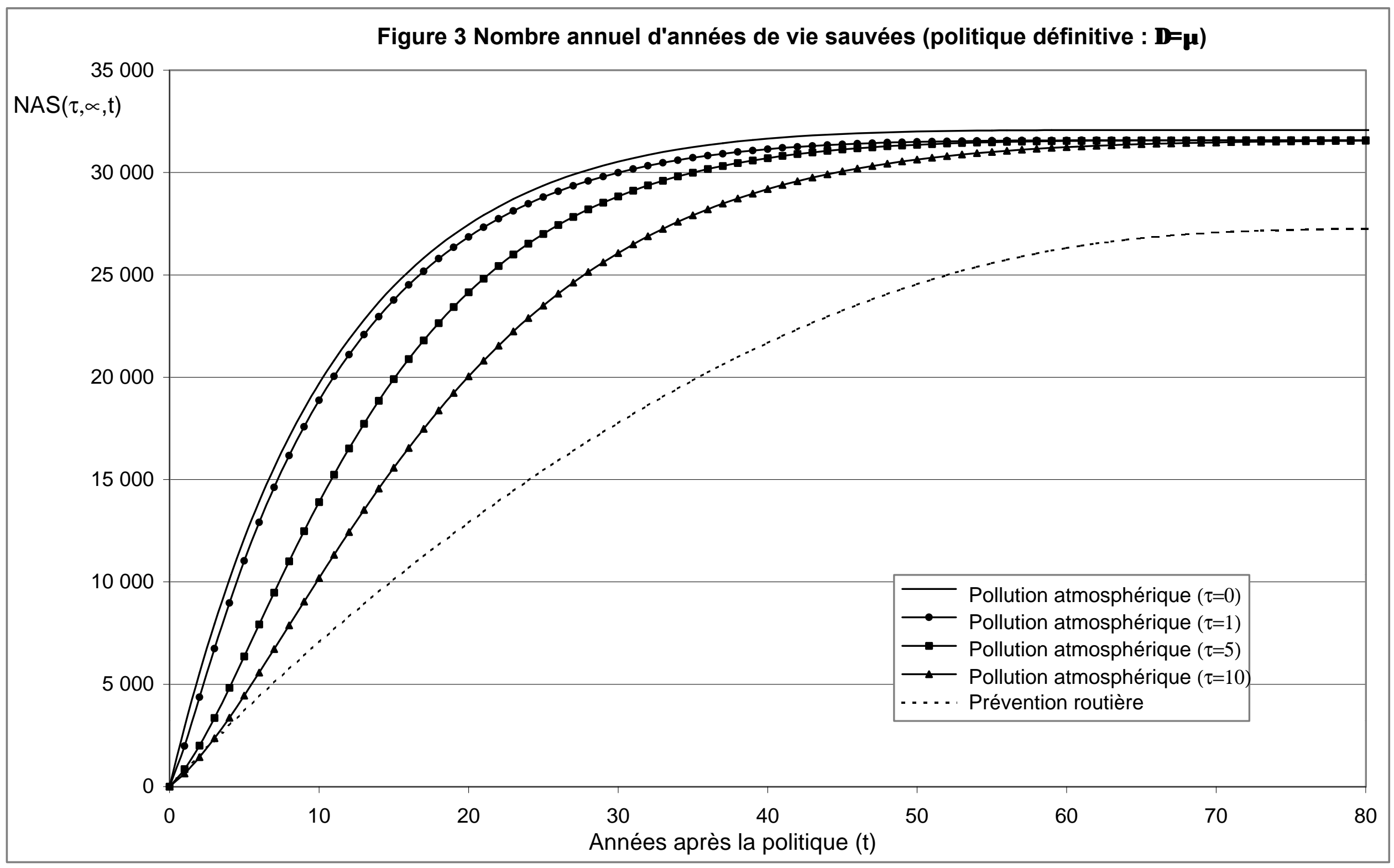




\section{Figure 4 Répartition des décès par âge}

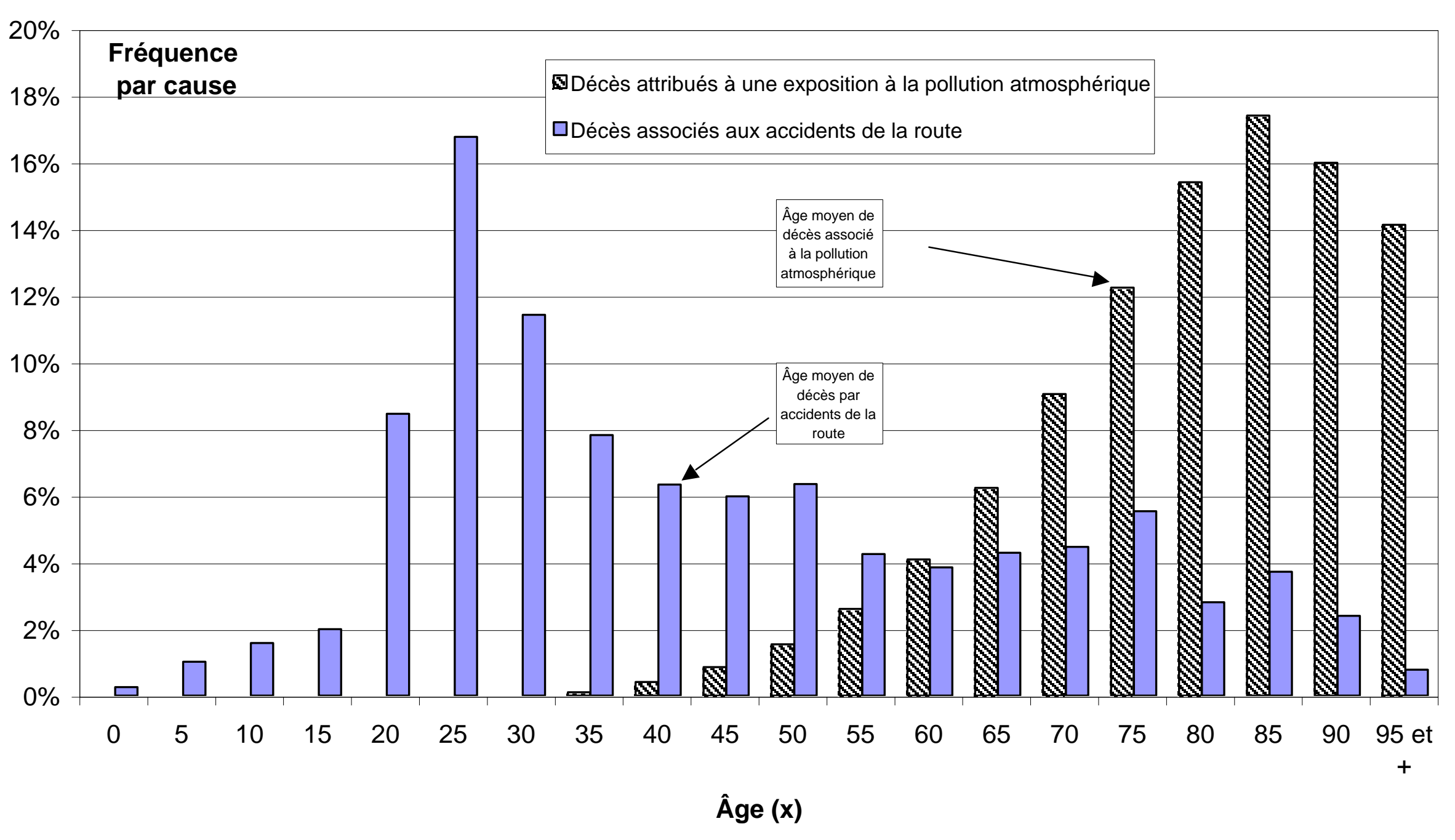

